\title{
An analogue of the Hardy theorem for the Cartan motion group
}

\author{
By Masaaki EGUChI, $\left.{ }^{*}, * *\right)$ Shin KolzUmI, $\left.{ }^{*)}, * * *\right)$ and Keisaku Kumahara $\left.* * * *\right)$ \\ (Communicated by Heisuke HIRONAKA, M. J. A., Dec. 14, 1998)
}

1. Introduction. The aim of this note is to prove an analogue of the Hardy theorem for the Cartan motion group. In the case of the Euclidean space, various forms of the uncertainty principle between a function and its Fourier transform are known. One of such theorems is known as the Hardy theorem. The Hardy theorem (cf. [1, pp. 155-158]) asserts that if a measurable function $f$ on $\boldsymbol{R}$ satisfies $|f| \leq C \exp \left\{-a x^{2}\right\}$ and $|\hat{f}|$ $\leq C \exp \left\{-b y^{2}\right\}$ then $f=0$, or $f$ is a constant multiple of $\exp \left\{-a x^{2}\right\}$, or there are infinitely many such $f$ according as $a b>1 / 4$, or $a b=1 / 4$, or $a b<1 / 4$. (Here we take $\hat{f}(y)=(1 / \sqrt{2 \pi}$ ) $\int_{-\infty}^{\infty} f(x) \exp \{\sqrt{-1} x y\} d x$ as the definition of the Fourier transform of $f$.)

Recently A. Sitaram and M. Sundari [5] generalized this theorem to the cases of the semisimple Lie groups with one conjugacy class of Cartan subgroups, the Riemannian symmetric spaces and $S L(2, \boldsymbol{R})$. And also M. Sundari [6] showed the Hardy theorem for the Euclidean motion group. By the way, in the case of the Cartan motion group, K. Kumahara [4] defined the Fourier transform by using the representation $\pi_{\xi}$ induced from $\xi \in \mathfrak{p}^{*}$ and studied the images of some function spaces under the Fourier transform. By using estimates of matrix elements of $\pi_{\xi}$ and applying a similar argument to $[5,6]$, we can get an analogue of the Hardy theorem for the Cartan motion group.

2. Notation and preliminaries. The standard symbols $\boldsymbol{Z}, \boldsymbol{R}$ and $\boldsymbol{C}$ shall be used for the integers, the real numbers and the complex num-

*) Partly supported by the Grants-in-Aid for Scientific Research (No. 09640190).

**) Faculty of Integrated Arts and Sciences, Hiroshima University, 1-7-1 Kagamiyama, HigashiHiroshima 739-8521.

***) Faculty of Information and Management, Onomichi Junior College, 1600 Hisayamada-cho, Onomichi, Hiroshina 722-0021.

****) The University of the Air, 2-11 Wakaba, Mihama-ku, Chiba 261-8586. bers. If $V$ is a vector space over $\boldsymbol{R}, V_{c}, V^{*}$ and $V_{c}^{*}$ denote its complexification, its real dual and its complex dual, respectively. For $v \in V_{c}, \Re v$, $\Im v$ and $\bar{v}$ denote its real part, its imaginary part and its complex conjugate, respectively. For a topological space $S$ and a subset $T$ of $S, \mathrm{Cl}(T)$ denotes the closure of $T$ in $S$. For a Lie group $L$, $\hat{L}$ denotes the set of equivalence classes of irreducible unitary representations of $L$.

Let $G_{0}$ be a connected semisimple Lie group with finite center and $\mathfrak{g}$ its Lie algebra. We fix a maximal compact subgroup $K$ of $G_{0}$ and denote by $\theta$ the corresponding Cartan involution. We set $\mathfrak{p}=\{X \in \mathfrak{g}: \theta X=-X\}$. Let $G_{0}=K A N$ and $\mathfrak{g}=\mathfrak{f}+\mathfrak{a}+\mathfrak{n}$ be Iwasawa decompositions of $G_{0}$ and $\mathfrak{g}$, respectively. We denote by $\langle\cdot, \cdot\rangle$ the Killing form of $\mathrm{g}$ and put $\|X\|^{2}=-\langle X, \theta X\rangle$. We also use the same symbols $\langle\cdot, \cdot\rangle$ and $\|\cdot\|$ the bilinear form and the norm on $\mathfrak{p}^{*}$ coming from those on $\mathfrak{p}$. In the notation introduced above, the semidirect product $G=K \ltimes \mathfrak{p}$ is called the Cartan motion group. For any $g \in G$, we write $g=(k(g), X(g))$, where $k(g) \in K$ and $X(g) \in \mathfrak{p}$. If $k \in K$ and $X \in \mathfrak{p}$, we simply write $k$ for $(k, 0)$ and $X$ for $(e, X), e$ being the unit element in $K$. With these notation, we have (2.1) $k\left(g^{-1}\right)=k(g)^{-1}, \quad X\left(g^{-1}\right)=-\operatorname{Ad}(k(g))^{-1} X(g)$, $k\left(g_{1} g_{2}\right)=k\left(g_{1}\right) k\left(g_{2}\right), \quad X\left(g_{1} g_{2}\right)=\operatorname{Ad}\left(k\left(g_{1}\right)\right) X\left(g_{2}\right)+X\left(g_{1}\right)$, for $g, g_{1}, g_{2} \in G$.

Let $M$ denote the centralizer of $\mathfrak{a}$ in $K$. For $g$ $\in G$ and $\xi \in \mathfrak{p}^{*}$, we define $g \xi \in \mathfrak{p}^{*}$ by $g \xi(X)$ $=\xi\left(\operatorname{Ad}(k(g))^{-1} X\right),(X \in \mathfrak{p})$. Let $\mathfrak{a}^{+}$and $\mathfrak{a}^{*+}$ be the positive Weyl chambers in $\mathfrak{a}$ and $\mathfrak{a}^{*}$, respectively. For $X \in \mathfrak{p}$, we choose $X^{+}$in $\mathrm{Cl}\left(\mathfrak{a}^{+}\right)$such that $X^{+} \in \operatorname{Ad}(K) X \cap \operatorname{Cl}\left(\mathfrak{a}^{+}\right)$. And if $\xi \in \mathfrak{p}^{*}$, we define $\xi^{+} \in \mathrm{Cl}\left(\mathfrak{a}^{*+}\right)$ by a similar way to the case of $\mathfrak{p}$. Let $g \in G$. If we pick $k_{1}, k_{2} \in K$ such that $\operatorname{Ad}\left(k_{1}\right) X(g)^{+}=X(g)$ and $k_{2}=k_{1}^{-1} k(g)$, then $g$ is written as $g=k_{1} X(g)^{+} k_{2}$. Thus we have the decomposition $G=K \mathrm{Cl}\left(\mathrm{a}^{+}\right) K$.

Finally let $d k$ be the Haar measure on $K$ normalized as $\int_{K} d k=1$. The Killing form induces Euclidean measures on $\mathfrak{p}$ and $\mathfrak{p}^{*}$. We nor- 
malize them by multiplying $(2 \pi)^{-\mathrm{dimp} / 2}$ and denote them by $d X$ and $d \xi$, respectively. Then $d g$ $=d k d X$ is a Haar measure on $G$. Under the decomposition $G=K \mathrm{Cl}\left(\mathfrak{a}^{+}\right) K$, the following integral formula holds (cf. [3, p. 195]).

$$
\text { (2.2) } \begin{aligned}
& \int_{G} f(g) d g \\
= & \int_{K / M} \int_{K} \int_{\mathfrak{a}_{+}} f\left(k_{1} H k_{2}\right) \prod_{\alpha \in \Sigma+} \alpha(H)^{m \alpha} d k_{1} d k_{2} d H .
\end{aligned}
$$

Here $\Sigma^{+}$denotes the set of all positive restricted roots and $m_{\alpha}$ denotes the multiplicity of $\alpha \in \Sigma^{+}$.

3. The Fourier transform on $\boldsymbol{G}$. In this note, according to $[2,4]$, we shall define the Fourier transform on $G$ by using the representations induced from the characters of $\mathfrak{p}$ instead of using the irreducible unitary representations for $G$. Let $\xi \in \mathfrak{p}^{*}$. Define the action $\pi_{\xi}$ of $G$ on $L^{2}(K)$ by

$$
\text { (3.1) } \begin{aligned}
& \left(\pi_{\xi}(g) \varphi\right)(k) \\
= & e^{\sqrt{-1} \xi\left(\operatorname{Ad}(k)^{-1} X(g)\right)} \varphi\left(k(g)^{-1} k\right),\left(\varphi \in L^{2}(K)\right) .
\end{aligned}
$$

Then $\left(\pi_{\xi}, L^{2}(K)\right)$ is a (reducible) unitary representation for $G$. It is to be noted that any irreducible unitary representation for $G$ is contained in $\pi_{\xi}$ for some $\xi \in \mathfrak{p}^{*}$ as an irreducible component. Let $R$ be the right regular representation of $K$ on $L^{2}(K)$. Then $R_{k} \pi_{\xi}(g)=\pi_{k \xi}(g) R_{k}$ for $k \in$ $K$. For $f \in L^{1}(G)$, we define its Fourier transform $\bar{f}(\xi)$ by

$$
\hat{f}(\xi)=\int_{G} f(g) \pi_{\xi}(g) d g .
$$

If $f \in L^{1}(G) \cap L^{2}(G)$, then the following Parseval formula holds (cf. [4]).

$$
\int_{G}|f(g)|^{2} d g=\int_{\mathfrak{p}^{*}}\|\hat{f}(\xi)\|_{H S}^{2} d \xi,
$$

where $\|\cdot\|_{H S}$ denotes the Hilbert-Schmidt norm.

In the following, for all $\tau \in \hat{K}$, we fix a representative of $\tau$ and by abuse of notation, write $\tau$ for it again. Since $\left.\pi_{\xi}\right|_{K}$ is the left regular representation of $K$ on $L^{2}(K)$, it follows from the Peter-Weyl theorem that

$$
\left.\pi_{\xi}\right|_{K}=\sum_{\tau \in \widehat{K}} d(\tau) \tau, L^{2}(K)=\sum_{\tau \in \widehat{K}} V_{\tau}^{*} \otimes V_{\tau} .
$$

Here $V_{\tau}$ denotes the representation space of $\tau$ and $d(\tau)=\operatorname{dim} V_{\tau}$. If $T \otimes v \in V_{\tau}^{*} \otimes V_{\tau}$, we set $\varphi_{T} \otimes_{v}(k)=T\left(\tau(k)^{-1} v\right) \in C^{\infty}(K)$. Let $g \in G$ and define

$$
\begin{aligned}
& \sigma(g)=\|X(g)\|, \\
& \Phi_{\xi}(g)=\int_{K} e^{\sqrt{-1} \xi\left(\operatorname{Ad}(k)^{-1} X(g)\right)} d k .
\end{aligned}
$$

Let $\tau_{1}, \tau_{2} \in \hat{K}$. We write $\mathscr{V}=\operatorname{Hom}_{C}\left(V_{\tau_{2}}, V_{\tau_{1}}\right)$ and denote by $\langle\cdot, \cdot\rangle_{\mathscr{V}}$ and $\|\cdot\|_{\mathscr{V}}$ the canonical inner product and the corresponding norm on $\mathscr{V}$ respectively, that is, for $S, T \in \mathscr{V},\langle S, T\rangle_{\mathscr{V}}=$ $\operatorname{tr}\left(T^{*} S\right)$ and $\|T\|_{\mathscr{V}}^{2}=\langle T, T\rangle_{\mathscr{V}}, T^{*}$ being the adjoint operator of $T$. For $T \in \mathscr{V}$, we set

(3.6) $E(T, \xi, g)$

$$
=\int_{K} \tau_{1}(k) T \tau_{2}\left(k^{-1} k(g)\right) e^{\sqrt{-1} \xi\left(\operatorname{Ad}(k)^{-1} X(g)\right)} d k .
$$

We first remark (cf. [3, p. 424]) that if $\xi \in \mathfrak{p}_{c}^{*}$ then

(3.7)

$$
\begin{aligned}
& \left|\Phi_{\xi}(g)\right| \\
& =\left|\Phi_{\xi}\left(X(g)^{+}\right)\right| \leq \int_{K} e^{-\Im \mathfrak{\xi}\left(\operatorname{Ad}(k)^{-1} X(g)^{+}\right)} d k \\
& =\int_{K} e^{-k(\Im \xi \xi)\left(X(g)^{+}\right)} d k \leq e^{(\mathfrak{g})^{+}\left(X(g)^{+}\right)},
\end{aligned}
$$

and thus the function $\xi \mapsto \Phi_{\xi}(g)$ makes sense for all $\xi \in \mathfrak{p}_{c}^{*}$. We also have for $k, k_{1}, k_{2} \in K, \xi \in$ $\mathfrak{p}_{c}^{*}$ and $g \in G$ that

$$
\begin{gathered}
\sigma\left(k_{1} g k_{2}\right)=\sigma(g), \quad \sigma\left(g^{-1}\right)=\sigma(g), \\
\Phi_{\xi}\left(k_{1} g k_{2}\right)=\Phi_{\xi}(g), \quad \Phi_{\xi}\left(g^{-1}\right)=\Phi_{\xi}(g), \\
E\left(T, \xi, k_{1} g k_{2}\right)=\tau_{1}\left(k_{1}\right) E(T, \xi, g) \tau_{2}\left(k_{2}\right), \\
E(T, \xi, g) *=E\left(T^{*}, \xi, g^{-1}\right) \\
E(T, k \xi, g)=E\left(\tau_{1}(k)^{-1} T \tau_{2}(k), \xi, g\right) .
\end{gathered}
$$

Moreover, we can easily get the following lemma.

Lemma 3.1. Retain the above notation. Let $T$ $\in \mathscr{V}$ and $T_{i} \otimes v_{i} \in V_{\tau_{i}}^{*} \otimes V_{\tau_{i}},(i=1,2)$.

(1) The function $\xi \mapsto E(T, \xi, g)$ can be extended to a holomorphic function on $\mathfrak{p}_{c}^{*}$.

(2) If $\xi \in \mathfrak{p}_{c}^{*}$ then

$\|E(T, \xi, g)\|_{\mathscr{V}} \leq\|T\|_{\mathscr{V}} e^{(\mathfrak{g} \xi)^{+}\left(X(g)^{+}\right)}$.

(3) If $\xi \in \mathfrak{p}^{*}$, then $\left\langle\pi_{\xi}(g) \varphi_{T_{2} \otimes_{v_{2}}}, \varphi_{T_{1}} \otimes_{v_{1}}\right\rangle_{L^{2}(K)}=\left\langle E\left(T_{1}^{*} T_{2}, \xi, g\right) v_{2}, v_{1}\right\rangle_{V \tau_{1}}$.

4. The main theorem. We first note that a measurable function $f$ on $G$ is said to be exponentially decreasing if $f$ satisfies the following condition :

$$
\text { ess. } \sup e^{r \sigma(g)}|f(g)|<\infty,
$$

for all $r \geq 0$. Such functions belong to $L^{p}(G)$ for all $1 \leq p \leq \infty$ and all the matrix elements $\left\langle\hat{f}(\xi) \varphi_{T_{2}} \otimes_{v_{2}}, \varphi_{T_{1}} \otimes_{v_{1}}\right\rangle_{L^{2}(K)}$ are holomorphic on $\mathfrak{p}_{c}^{*}$ (cf. [2]). To prove the Hardy theorem, we need the following lemma of A. Sitaram and M. Sundari.

Lemma 4.1 [5, Lemma 2.1]. Let $h$ be an entire function on $C^{n}$ such that

$|h(z)| \leq C e^{a|| z||^{2}},\left(z \in C^{n}\right),|h(t)| \leq C e^{-a|| t||^{2}},\left(t \in \boldsymbol{R}^{n}\right)$, for some $a>0$ and $C>0$. Then $h(z)=$ const. $\exp \left\{-a\left(z_{1}^{2}+\cdots+z_{n}^{2}\right)\right\}$.

Our aim is to prove the following theorem.

Theorem 4.2 (the Hardy theorem). Let $f$ be a 
measurable function on $G$ such that

$$
|f(g)| \leq C e^{-a \sigma(g)^{2}},\|\hat{f}(\xi)\|_{H S} \leq C e^{-b|| \xi||^{2}},
$$

for $C>0, a>0$ and $b>0$. If $a b>\frac{1}{4}$, then $f=$ 0 (a.e.).

Proof. Since $f$ is exponentially decreasing, $f$ $\in L^{1}(G) \cap L^{2}(G)$ and thus the Parseval formula holds for $f$. Let $\tau_{1}, \tau_{2} \in \hat{K}$ and choose $T_{i} \in V_{\tau_{i}}^{*}$ and $v_{i} \in V_{\tau_{i}},(i=1,2)$ so that $\left\|T_{i}\right\|_{V_{\tau_{i}}^{*}}=\left\|v_{i}\right\|_{V_{\tau_{i}}}$ $=1$. We have from Lemma 3.1 (3) and (2.2) that

$$
\begin{aligned}
& \left\langle\hat{f}(\xi) \varphi_{T_{2}} \otimes_{v_{2}}, \varphi_{T_{1}} \otimes_{v_{1}}\right\rangle_{L^{2}(K)} \\
= & \int_{G} f(g)\left\langle E\left(T_{1}^{*} T_{2}, \xi, g\right) v_{2}, v_{1}\right\rangle_{V_{\tau_{1}}} d g \\
= & \int_{K / M} \int_{K} \int_{a^{+}} f\left(k_{1} H k_{2}\right)\left\langle E\left(T_{1}^{*} T_{2}, \xi, k_{1} H k_{2}\right) v_{2}, v_{1}\right\rangle_{V_{\tau_{1}}} \\
& \Pi \alpha(H)^{m_{\alpha}} d k_{1} d k_{2} d H .
\end{aligned}
$$

We have from the assumption of $f$ and Lemma 3.1 (2) that

$$
\begin{aligned}
& \text { (4.3) }\left|f\left(k_{1} H k_{2}\right)\right| \leq C e^{-a \sigma\left(k_{1} H k_{2}\right)^{2}}=C e^{-a|| H||^{2}}, \\
& \left|\left\langle E\left(T_{1}^{*} T_{2}, \xi, k_{1} H k_{2}\right) v_{2}, v_{1}\right\rangle_{V_{\tau_{1}}}\right| \leq e^{(\Im \xi)^{+}(H)},
\end{aligned}
$$
and hence

$$
\leq \operatorname{vol}(K / M) \int_{a_{+}} C e^{-a|| H \|^{2}} e^{(\Im \xi)^{+}(H)} \prod_{\alpha \in \Sigma+} \alpha(H)^{m \alpha} d H,
$$

where $\operatorname{vol}(K / M)=\int_{K / M} d k$.

$$
\text { Choose } 0<a^{\prime}<a \text { so that } a^{\prime} b>\frac{1}{\Lambda} \text {, then }
$$

$e^{-a|| H \|^{2}} \Pi_{\alpha \in \Sigma^{+}} \alpha \quad(H)^{m \alpha} \leq C^{\prime} e^{-\left.a^{\prime}|| H\right|^{2}}$ for some constant $C^{\prime}>0$. Since $(\Im \xi)^{+} \in \mathrm{Cl}\left(a^{*+}\right)$, there exists $H_{\xi} \in \mathrm{Cl}\left(\mathfrak{a}^{+}\right)$such that $(\Im \xi)^{+}(H)=\langle H$, $H_{\xi}>$ for $H \in \mathfrak{a}^{+}$. Taking into account $\left\|H_{\xi}\right\|=$ $\left\|(\mathfrak{g} \xi)^{+}\right\|=\|\mathfrak{\xi}\| \leq\|\xi\|$, we see that

$$
\begin{aligned}
& \left|\left\langle\hat{f}(\xi) \varphi_{T_{2}} \otimes_{v_{2}}, \varphi_{T_{1}} \otimes_{v_{1}}\right\rangle_{L^{2}(K)}\right| \leq C C^{\prime} \operatorname{vol}(K / M) \int_{a^{+}} e^{-a^{\prime}|| H \|^{2}+\langle H, H \xi\rangle} d H \\
= & C C^{\prime} \operatorname{vol}(K / M) e^{\frac{1}{4 a^{\prime}}|| H_{\xi} \|^{2}} \int_{a^{+}} e^{-a^{\prime}\left\langle H-\frac{1}{2 a^{\prime}} H \xi, H-\frac{1}{2 a^{\prime}} H_{\xi}\right\rangle} d H \\
\leq & C C^{\prime} \operatorname{vol}(K / M) e^{\frac{1}{4 a^{\prime}}|| \xi||^{2}} \int_{a^{+}} e^{-a^{\prime}|| H \|^{2}} d H .
\end{aligned}
$$

Since the integral appeared in the last expression in (4.5) had to be bounded, we can find a constant $C_{1}>0$ such that

$$
\text { (4.6) }\left|\left\langle\hat{f}(\xi) \varphi_{T_{2}} \otimes_{v_{2}}, \varphi_{T_{1}} \otimes_{v_{1}}\right\rangle_{L^{2}(K)}\right| \leq C_{1} e^{\frac{1}{4 a^{\prime}}|| \xi||^{2}} \text {, }
$$
for all $\xi \in \mathfrak{p}_{c}^{*}$.

On the other hand, it follows from the assumption of $\hat{f}$ that
(4.7) $\left|\left\langle\hat{f}(\xi) \varphi_{T_{2} \otimes_{v_{2}}}, \varphi_{T_{1} \otimes_{v_{1}}}\right\rangle_{L^{2}(K)}\right| \leq C e^{-b|| \xi \|^{2}} \leq C e^{-\frac{1}{4 a^{\prime}}\|\xi\|^{2}}$, for all $\xi \in \mathfrak{p}^{*}$. Applying Lemma 4.1 together with (4.6) and (4.7), we can find a constant $C_{2}$ such that for $\xi \in \mathfrak{p}^{*}$,

$$
\text { (4.8) }\left\langle\hat{f}(\xi) \varphi_{T_{2}} \otimes_{v_{2}}, \varphi_{T_{1}} \otimes_{v_{1}}\right\rangle_{L^{2}(K)}=C_{2} e^{-\left.\frac{1}{4 a^{\prime}}|| \xi\right|^{2}} .
$$

Consequently we have

$$
\left|C_{2}\right| e^{\left(b-\frac{1}{4 a^{\prime}}\right)|| \xi||^{2}} \leq C
$$

and, since $b-\frac{1}{4 a^{\prime}}>0$, this is impossible unless $C_{2}=0$. So

(4.10) $\left\langle\hat{f}(\xi) \varphi_{T_{2}} \otimes_{v_{2}}, \varphi_{T_{1} \otimes_{v_{1}}}\right\rangle_{L^{2}(K)}=0$,

and the Parseval formula yields

(4.11) $\int_{G}|f(g)|^{2} d g=\int_{p^{*}}\|\hat{f}(\xi)\|_{H S}^{2} d \xi=0$.

Thus we have $f=0$ (a.e.).

Remark. In [2], M. Eguchi, K. Kumahara and Y. Muta studied the characterizations of the Schwartz spaces for the motion groups. In their paper, by the motion group is meant the semidirect product $K \ltimes V$ of a real vector space $V$ and a connected compact Lie group $K$ acting orthogonally on $V$. For such groups, if we define the Fourier transform by using the representations induced from the characters of $V$, we can get the Hardy theorem for the motion group by applying a similar argument to this note.

\section{References}

[1] H. Dym and H. P. McKean: Fourier series and integral. Academic Press, New York (1972).

[2] M. Eguchi, K. Kumahara, and Y. Muta: A subspace of Schwartz space on motion group. Hiroshima Math. J., 10, 691-698 (1980).

[3] S. Helgason: Groups and Geometric Analysis. Academic Press, New York (1984).

[4] K. Kumahara: Fourier transforms on the Cartan motion group. Proc. Japan Acad., 50, 433-435 (1974).

[5] A. Sitaram and M. Sundari: An analogue of Hardy's theorem for very rapidly decreasing functions on semi-simple Lie groups. Pacific J. Math. 177, 187-200 (1997).

[6] M. Sundari: Hardy's theorem for the $n$-dimensional Euclidean motion group. Proc. Amer. Math. Soc., 126, 1199-1204 (1998). 